\title{
Doble filicidio por suicidio ampliado (frustrado) de un sujeto afecto de depresión mayor psicótico y trastorno de la personalidad dependiente
}

\section{Double filicide by extended (frustrated) suicide of a subject affected by psychotic major depression and dependent personality disorder}

\author{
DOI: $10.46932 / \mathrm{sfjdv2n4-042}$
}

Received in: May 1st, 2021

Accepted in: Jun 30th, 2021

\author{
Dr. Bernat-Noël Tiffon Nonis \\ Universitat Abad Oliba-CEU. Calle de Bellesguard, no30. 08022 Barcelona (Spain). \\ E-mail: btiffonn@uao.es
}

\section{RESUMEN}

Se ilustra un caso de suicido ampliado frustrado, en el que la perpetradora no logra consumar su propio suicidio, pero habiendo perpetrado el asesinato de sus propios 2 hijos a causa del sufrimiento de su trastorno depresivo mayor grave con sintomatología psicótica y rasgos de personalidad dependientes de la personalidad. Se ilustra el caso con las pruebas psicométricas administradas y se analiza la psicometría forense del caso.

Palabras chave: suicidio ampliado, asesinato, trastorno depresivo mayor psicótica,

\section{ABSTRACT}

A case of frustrated extended suicide is illustrated, in which the perpetrator fails to consummate her own suicide, but having perpetrated the murder of her own 2 children because of the suffering of her major depressive disorder with psychotic symptomatology and personality-dependent personality traits. The case is illustrated with the psychometric tests administered and the forensic psychometrics of the case are analyzed.

Key words: extended suicide, murder, psychotic major depressive disorder.

\section{INTRODUCCIÓN.}

En términos generales, existen pocos o nulos estudios de investigación y/o técnicas de evaluación psicológica en cuanto a la capacidad predictiva de un comportamiento agresivo del alcance de un suicidio ampliado (Tiffon y González-Fernández, 2021). De modo que, cuando un padre o una madre dan muerte a su propio hijo, se conoce como filicidio. Dicha situación criminológica siempre origina un gran impacto social, jurídico y mediático (Tiffon, 2017, 2021).

La palabra «filicidio» proviene del latín filuis, hijo, y caedére, matar, y designa la muerte del propio hijo por parte del padre o la madre. Cuando uno de los progenitores mata a su bebé recién nacido comete un homicidio que se etiqueta como infanticidio. En general, filicidio queda reservado para niños con rangos de edades entre 1 y 18 años, subdividiéndose en tipo temprano cuando incluye las edades de 
1 a 12 años y tipo tardío más de 13 años (Estupiñán et al.; 2016; Tiffon, 2017 y Tiffon y GonzálezFernández, 2021).

En términos generales, en los delitos de sangre perpetrados por sujetos con impulsividad patológica no existen condicionantes para una atenuación de la pena, de no concurrir con algún otro tipo de trastorno mental de naturaleza grave (Tiffon y González-Fernández, 2021 ${ }^{\mathrm{b}}$ ). en el presente caso, la informada presenta dicho trastorno afectivo pero que no alcanza ni reviste una circunstancia modificativa de la responsabilidad criminal de notoria importancia para el sistema judicial español. No obstante, si existe un componente psicótico en su trastorno depresivo mayor que afectase sus capacidades cognitivas y/o volitivas, de modo que el contenido de pensamiento psicótico-delirante debe contextualizarse dentro de los límites de lo posible, con una coherencia suficiente como para mostrarse aceptable. Aunque este fenómeno no excluye al sexo masculino, suele ser más frecuente que se manifieste en población de sexo femenino (Tiffon, 2021).

\section{EL CASO.}

Se trata de una mujer de origen sudamericano, de unos 30 años, separada y madre de dos hijos que, en el momento del óbito de los menores presentaban la edad de 11 y de 9 años. El objeto del informe era valorar el estatus psíquico y mental de la informada y establecer una relación de compatibilidad retrospectiva de su conducta al momento de perpetrar los hechos luctuosos. La victimaria sufría de trastorno depresivo mayor y presentaba rasgos de personalidad de base de tipo dependiente; asimismo, la misma pasaba por una serie de factores psicosociales adversos que agravaban su estado anímico y psicoemocional que agravaba su condición psicopatología de base y por el que decidiera asesinar a sus dos hijos en un contexto patológico de suicidio ampliado; sien en este caso, frustrado a ser rescatada por la figura de su exmarido. El íter criminis perpetrado fue someter a los menores a la ingesta de pastillas depresoras del sistema nervioso central con el fin de adormecerlos para, seguidamente, proceder a ahogarlos en la bañera de su domicilio. La informada presentó una aguda y crítica alteración psicoemocional momentos previos a la comisión del suceso, como efecto de los sucesos precursores que experimentó como perjudiciales contra su persona e integridad física (Tiffon, 2021). A la luz de lo anteriormente descrito, la conducta delictivo-criminológica fue el producto de un conjunto de factores psicopatológicos de base (añadidos a factores psicosociales adversos) que actuaron como condicionantes previos en la materialización conductual anteriormente descrita (Tiffon, 2021).

La cronología de hechos: el mismo día que se cometen los hechos luctuosos, la informada ingresa en Servicio de Urgencias de un Hospital de Barcelona y permanece durante 3 días antes de proceder su ingreso en el Centro Penitenciario. Al cabo de 3 días de su ingreso en el hospital, fue explorada por los 
Médicos Forenses y, a continuación, fue trasladada e ingresada en la Unidad de Agudos de la Unidad Hospitalaria Psiquiátrica Penitenciaria (UHPP) del Centro Penitenciario. Allí permanecería ingresada 3 meses, para ser trasladada posteriormente al Centro Penitenciario de Mujeres.

\section{MÉTODO.}

Para la elaboración del presente caso se procedió a las siguientes fuentes de información:

1- Entrevistas clínico-periciales con la informada mantenida en el Centro Penitenciario de Mujeres durante 3 días.

2- Entrevista con el hermano de la informada.

3- Entrevistas telefónicas mantenidas con una amiga de la informada.

4- Estudio psicopatológico y psicométrico: MMPI-2, MCMI-III, Cuestionario Salamanca de Trastornos de Personalidad, 16 PF-5, Inventario de Evaluación de la Personalidad - PAI, Escala de Hamilton para la Depresión-HDRS-, Inventario de Ansiedad Estado-Rasgo-StateTrait Anxiety Inventory, STAI-, Escala de Hamilton para la Ansiedad -HARS-, Escala de Desesperanza de Beck -Hopelessness Scale, HS-, Escala de Riesgo Suicida de Plutchik -Risk of Suicide, RS-, Escala de Ideación Suicida (Scale for Suicide Ideation, SSI).

5- Estudio de la documentación clínico-jurídica aportada (Informe Psiquiátrico de la Unidad de Hospitalización Psiquiátrica Penitenciaria —UHPP—e Informe Médico-Forense).

\section{RESULTADOS.}

Los resultados obtenidos de las distintas pruebas psicológicas y psicopatológicas administradas son compatibles con la existencia de trastorno del estado del ánimo: Trastorno Depresivo Mayor de intensidad grave con sintomatología psicótica y tendencia suicida (ver puntuaciones clínica y estadísticamente significativas en las escalas pertenecientes a los trastornos depresivos y rasgos psicóticos del MCMI-III y del PAI).

Asimismo, las puntuaciones también sugieren la existencia de trastorno de la personalidad por dependencia (ver puntuaciones clínica y estadísticamente significativas en las escalas pertenecientes a los trastornos depresivos y rasgos psicóticos del MCMI-III, del PAI y del 16 PF-5). 
Figura 1: Resultados y perfil psicológico y psicopatológico del informado obtenido del Inventario Clínico Multiaxial de Millón (MCMI-III, versión 3 del año 2005).

\section{P Pearson}

\section{INVENTARIO CLÍNICO MULTIAXIAL DE MILLON-III}

VALIDEZ $=0 \quad$ INCONSISTENCIA $=7$

CÓDIGO DE PERSONALIDAD: - " $12 \mathrm{~A} 2 \mathrm{~B} * \underline{37} 8 \mathrm{~B} 6 \mathrm{~A}+8 \mathrm{~A} 6 \mathrm{~B}$ " $54 / /-^{* \pi}-{ }^{*} / /$

CÓDIGO DE SINDROME: A " D H $* / /-" \mathrm{CC} P \mathrm{PP} * \| /$

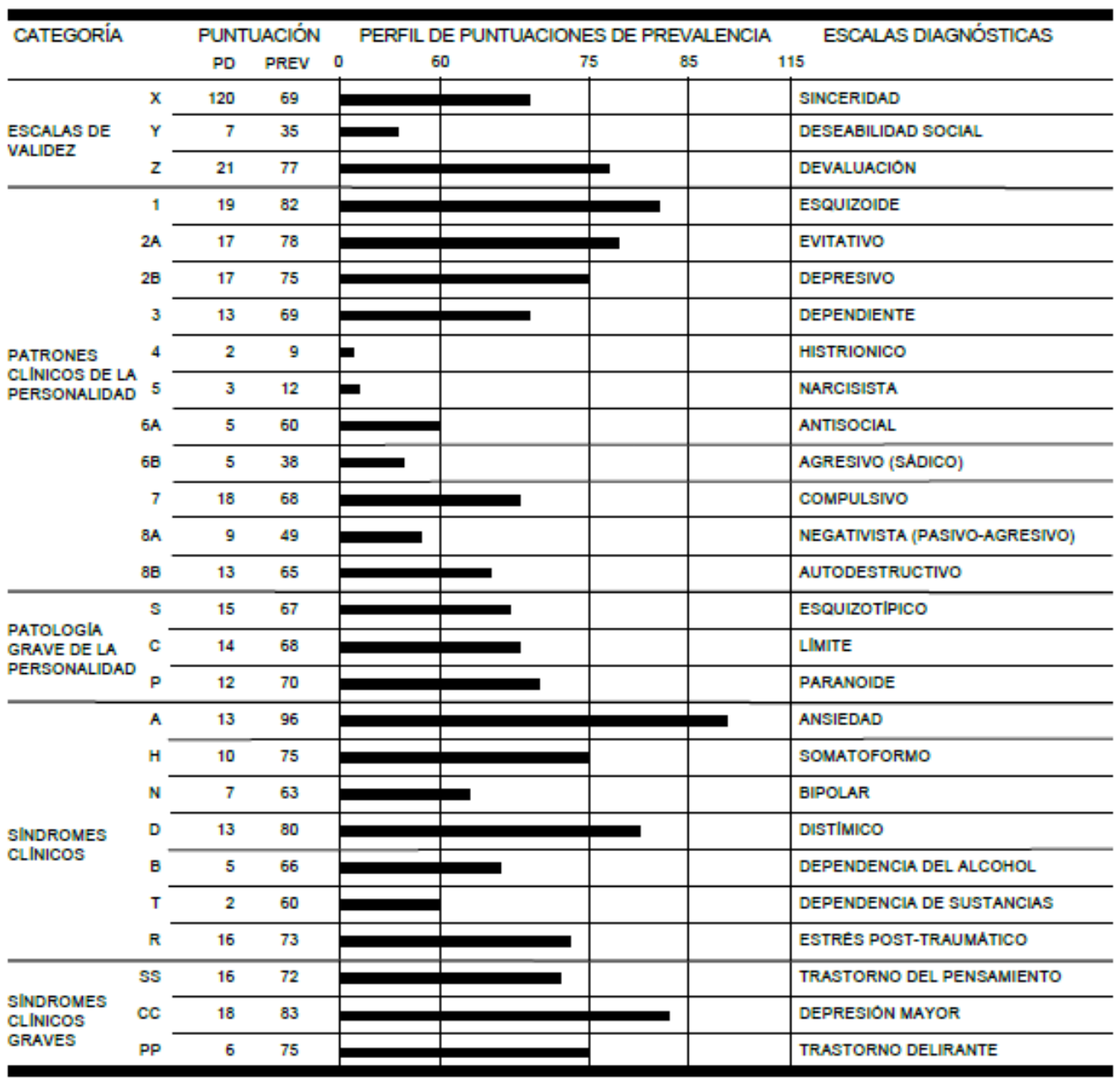


Figura 2a: Resultados y perfil psicológico y psicopatológico del informado obtenido del Inventario de Evaluación de la Personalidad (PAI) de Morey (2011).
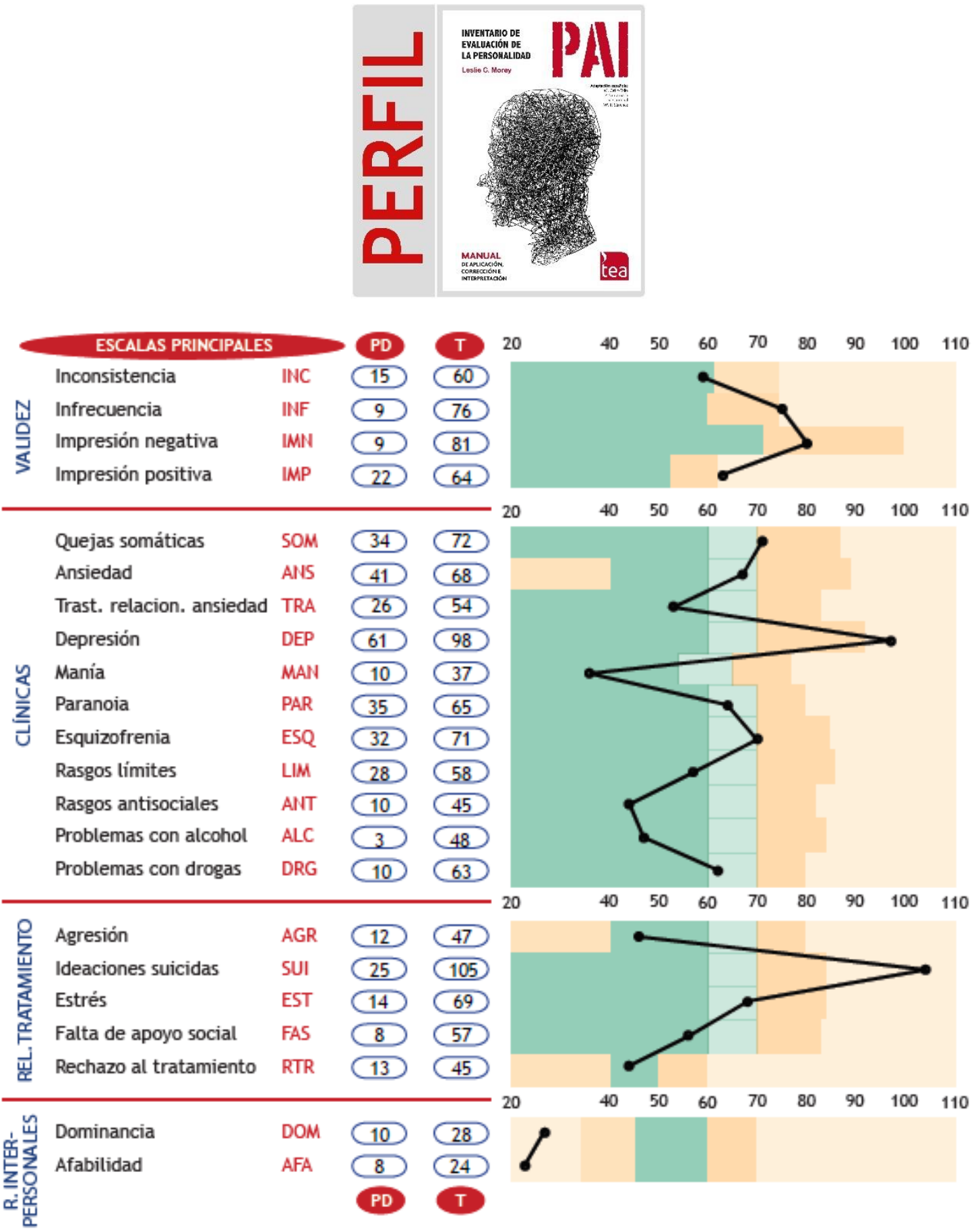
Figura 2b: Resultados y perfil psicológico y psicopatológico del informado obtenido del Inventario de Evaluación de la Personalidad (PAI) de Morey (2011).






\section{INDICES COMPLEMENTARIOS}

Inconsistencia al final del cuestionariolNC-F

Índice de simulación

$\operatorname{SIM}$

Función discriminante de Rogers FDR

Índice de defensividad

DEF

Función discriminante de Cashel FDC



40



6070

8090

100

110

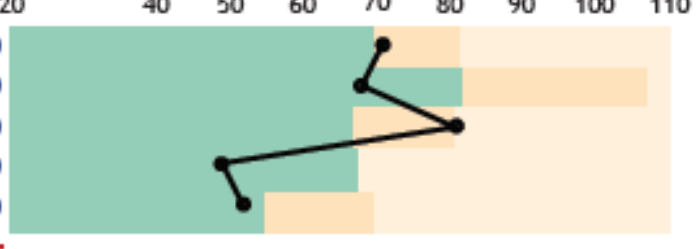

Índice potencial de suicidio IPS

Índice potencial de violencia IPV

Índice de dificultad de Tratamiento IDT



\section{西}

Índice est. problemas con el alcohol ALC-Est índice est. problemas con las drogas DRO-Est

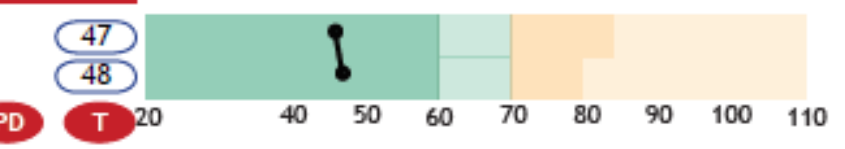

Figura 3: Resultados y perfil psicológico del informado obtenido de la prueba 16 Factores de Personalidad (16PF) de Cattel (1995).






\begin{tabular}{ll}
\hline & ESCALAS PRIMARIAS \\
\hline A & Afabilidad \\
B & Razonamiento \\
C & Estabilidad \\
E & Dominancia \\
F & Animación \\
G & Atención a las normas \\
H & Atrevimiento \\
I & Sensibilidad \\
L & Vigilancia \\
M & Abstracción \\
N & Privacidad \\
O & Aprensión \\
Q1 & Apertura al cambio \\
Q2 & Autosuficiencia \\
Q3 & Perfeccionismo \\
Q4 & Tensión \\
\hline & ESTILOS DE RESPUESTA
\end{tabular}


PD

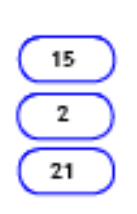

Ext Extraversión
Ans Ansiedad
Dur Dureza
Ind Independencia
AuC Auto-control

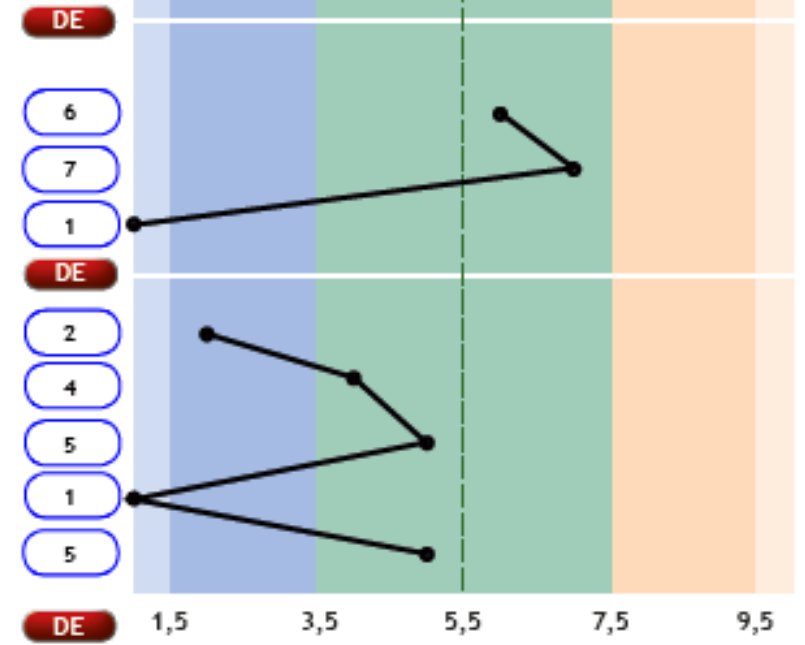

\section{DISCUSIÓN}

Según la documentación clínica obrante cercana a la perpetración de los hechos, la informada presenta compatibilidad con que, hacia el momento del suicidio ampliado frustrado, la misma presentaba:

a) Trastorno Depresivo Mayor de intensidad Grave (código F32.31 de la CIE-10-OMS).

b) Trastorno de la Personalidad Dependiente (código F60.7 de la CIE-10-OMS).

La informada experimentaba una serie de factores psicosociales adversos, tales como:

a) La vivencia de malos tratos físicos y verbales recibidos por parte de su cónyuge de manera continuada, así como de amenazas. 
b) Precariedad económica dada su imposibilidad de reincorporarse al mercado laboral, llegando a necesitar la ayuda de servicios sociales y religiosos.

c) Imposibilidad de retornar al país de origen por difícil situación pecuniaria y dada la necesidad de tratamiento médico-quirúrgico de su hija mayor, aquejada de displasia ósea.

d) Temor a perder la custodia de sus hijos dada la situación de elevada conflictividad psicosocial con relación a su exmarido y la incapacidad de conseguir una estabilidad laboral y económica.

Con relación a los presuntos hechos de autos, la informada presenta una estructura caracterial caracterizada por la existencia de rasgos de personalidad de base de dependencia emocional, baja autoestima y sumisión; éstos vinieron a fracturarse y a agravarse psico-emocionalmente presentando una situación de vulnerabilidad respecto a la patología emocional, interviniendo de manera continuada e interrelacionada los factores estresógenos y adversos externos anteriormente mencionados (grave problemática familiar, económica y laboral), desarrollándose un Trastorno Adaptativo Mixto Grave Ansioso-Depresivo que evolucionó progresivamente incubándose -a la par- en un Trastorno Depresivo Mayor de intensidad Grave con sintomatología psicótica.

Como factor de co-morbilidad (concomitancia de varias patologías) cabe señalar que en la fecha del día de autos experimentó una descompensación en forma de reacción de estrés emocional agudo, asociado a una intoxicación medicamentosa con hipnótico-sedantes, con un claro efecto sobre las funciones cognitivas superiores, con predeterminada intencionalidad autolítica, y con posteriores efectos disociativos no facilitadores al recuerdo posterior de dichos acontecimientos.

En este contexto anteriormente descrito, los presuntos hechos son compatibles con una situación de suicidio ampliado -tal como consta en el Informe de Asistencia de Urgencias de un Hospital-, siendo en el presente caso, frustrado, al no completarse con la defunción de la intencionante).

La concatenación de los factores patológicos anteriormente descritos vino a determinar una profunda afectación cognitiva durante la materialización de los presuntos hechos de autos. En este sentido se puede considerar como compatible que la capacidad analítico-reflexiva e intelectiva estuvo significativa y altamente afectada por la distorsión cognitiva provocada por los trastornos descritos, agravada por la ingesta e intoxicación de psicofármacos que afectaron la conciencia de esta y rompieron los mecanismos inhibitorios de su conducta (Tiffon, 2017).

\section{CONCLUSIONES.}

Los pacientes que sufren trastornos depresivos y que cursan con contenidos de pensamientos de muerte o ideación suicida tenían una menor confianza en sí mismos, una excesiva dependencia de otros y un elevado nivel autopunitivo en comparación con pacientes deprimidos sin pensamientos de muerte o 
suicidio (Fountoulakis K, Iacovides A, Fotiou F, et al., 2004). Para Joiner, Brown, y Wingate, (2005), la falta de esperanza es un correlato psicológico fundamental del suicidio. Un estudio sobre la falta de esperanza ha demostrado una correlación más fuerte entre esta, la ideación suicida y el suicidio que, entre la falta de esperanza y la depresión, y la depresión y el suicidio.

En la línea de lo anteriormente descrito, el presente caso viene a converger con lo descrito por los autores anteriormente mencionados, de modo que sujetos con trastorno depresivo mayor con rasgos de personalidad adolecidos de sintomatología de tipo psicótico-paranoide y que presentan una tendencia de personalidad de tipo dependiente-evitativo, suelen ser más frágiles o más vulnerables a las adversidades psicosociales, y por tanto, a la tendencia al pensamiento o conducta suicida.

La fragilidad de su estado psicoemocional, ya adolecida de por sí por los trastornos psicopatológicos anteriormente mencionados, hace que dicho colectivo de sujetos sea más sensibles o vulnerables (menos resilientes, según su grado de fragilidad psíquica) a los acontecimientos estresógenos. 


\section{REFERENCIAS}

- Cattell, R.B.; Cattell, A. K., Cattell, H.E.P. (1995). 16 PF-5. Cuestionario Factorial de Personalidad. TEA Ediciones. Madrid.

- $\quad$ Estupiñán Rodríguez N, Rodríguez Hernández E, Gallardo Sarmiento A, Jiménez Ramírez I, Bayona Santana DR, Villalonga Costa L. Filicidio: apuntes históricos y reflexión actual. revmedicaelectronica [Internet]. 2016 [citado 18 Jul 2021];, 38(3):[aprox. 7 p.]. Disponible en: http://revmedicaelectronica.sld.cu/index.php/rme/article/view/1480

- $\quad$ Fountoulakis K, Iacovides A, Fotiou F, et al. Neurobiological and psychological correlates of suicide attempts and thoughts of death in patients with major depression. Neuropsychobiology. 2004; 49:42-62

- $\quad$ Joiner, T.E.; Brown, J.S.; Wingate, L.R. The psychology and neurobiology of suicidal behavior. Annu. Rev. Psychol. 2005; 56:287-314.

- $\quad$ Millon, T.; Davis, R. y Millon, C. (2011 - [Ed. orig. TEA Ediciones, 2007]). «Inventario Clínico Multiaxial de Millon-III». Pearson Educación. Madrid.

- Morey, L.C. (2007). «PAI. Personality Assessment Inventory». Psychological Assessment Resources, Florida, EE.UU.

- $\quad$ Tiffon, B.N. (2015). “Los Crímenes de Perejil”. J.M. Bosch Editor. Barcelona.

- $\quad$ Tiffon, B.-N. (2016). “Archivos Delictivo-Criminológicos”. J.M. Bosch Editor. Barcelona.

- $\quad$ Tiffon, B.-N. (2017). “Atlas de Psicología Forense (Penal)”. J.M. Bosch Editor. Barcelona.

- Tiffon, B.-N. (2017). “A propósito de un caso de doble filicidio por suicidio ampliado frustrado”. Revista de Derecho y Proceso Penal, no. 48, págs. 335-349.

- $\quad$ Tiffon, B.-N. y Cols. (2019). “Atlas Práctico-Criminológico De Psicometría Forense (Volumen I): Asesinatos”. J.M. Bosch Editor. Barcelona.

- $\quad$ Tiffon, B.-N. y Cols. (2019). “Atlas Práctico-Criminológico De Psicometría Forense (Volumen II): Tentativas De Asesinatos”. J.M. Bosch Editor. Barcelona.

- $\quad$ Tiffon, B.-N. (2021). "Criminal and Forensic Psychology of a Case of Filicide by Decapitation of a Minor". Journal of Forensic Medicine. Case Series. Volume 6:3. ISSN: 2472-1026

- $\quad$ Tiffon, B.-N- (2021). "Murder induced deliriously by a "folie à deux". Vol. 2 No. 2 (2021): South Florida Journal of Development, Miami, v. 2, n. 2, apr./jun. 2021 ISSN: 2675-5459_Págs. 35603564.DOI: https://doi.org/10.46932/sfjdv2n2-191 
- $\quad$ Tiffon, B.-N. (2021). "Criminal personality in a trained elite military and assassination". Vol. 2 No. 2 (2021): South Florida Journal of Development, Miami, v. 2, n. 2, apr./jun. 2021 ISSN: 2675-5459 Págs. 3555-3559. DOI: https://doi.org/10.46932/sfjdv2n2-190

- $\quad$ Tiffon, B.-N. (2021) ${ }^{\text {d }}$ "Amok syndrome in a quadruple crime, acting out and pathological drive.". Vol. 2 No. 2 (2021): South Florida Journal of Development, Miami, v. 2, n. 2, apr./jun. 2021 ISSN: 26755459. DOI: https://doi.org/10.46932/sfjdv2n2-189

- $\quad$ Tiffon, B.-N. (2021). "Double filicide for extended suicide (frustrated) of a subject with major psychotic depression and dependent personality disorder". South Florida Journal of Development, Miami, v. 2, n. 3, Special Edition, jul. 2021._Págs. 4552-4562. DOI: https://doi.org/10.46932/sfjdv2n3-057

- $\quad$ Tiffon, B.-N. (2021). "Delusional psychotic disorder, substance use and murder with alevosia and cruelty". South Florida Journal of Development, Miami, v.2, n.3, p.4544-4551 Special Edition, jul. 2021. Págs. 4544-4550. DOI: https://doi.org/10.46932/sfjdv2n3-056

- $\quad$ Tiffon, B.-N. \& González-Fernández (2021). "Desperation In Major Serious Depressive Disorders And Extended Suicide Risk: A Case Of Double Filicide". Asean Journal Of Psychiatry, Vol. 22(S2), June 2021: 1-5. ISSN: 2231-7805

- $\quad$ Tiffon, B.-N. \& González-Fernández (2021). "Desperation In Major Serious Depressive Disorders And Extended Suicide Risk: A Case Of Double Filicide". Asean Journal Of Psychiatry, Vol. 22(S2), June 2021: 1-5. ISSN: 2231-7791

- $\quad$ Tiffon, B.-N. \& González-Fernández (2021). "Amok Syndrome. Qualified Perspectives on an Aggressive Reaction of Pathological Impulsiveness in The Perpetration of a Double Crime with a Firearm". ASEAN Journal of Psychiatry, Vol. 22(S2), June 2021: 1-5 ISSN: 2231-7805

- $\quad$ Tiffon, B.-N. \& González-Fernández (2021). "Amok Syndrome. Qualified Perspectives on an Aggressive Reaction of Pathological Impulsiveness in The Perpetration of a Double Crime with a Firearm". ASEAN Journal of Psychiatry, Vol. 22(S2), June 2021: 1-5. ISSN: 2231-7791

- $\quad$ Tiffon, B.-N. \& González-Fernández. J. (2021). "Cocaine Cravings, Borderline Personality Disorder \& Attempted Homicide". ASEAN Journal of Psychiatry, Vol. 22(5), July 2021; 1-4._ISNN: 22317791 - Págs. 1-4

- $\quad$ Tiffon, B.-N. \& González-Fernández, J. (2021). Alcohol Consumption and Intensification of Paranoid Traits in Attempted Homicide. ASEAN Journal of Psychiatry, Vol. 22(4), June 2021: 1-5. ISSN: 2231-7805. Págs. 1-5 